\title{
Latest Reactor Neutrino Oscillation Results from the Daya Bay Experiment
}

\section{Bedrich Roskovec ${ }^{a, *}$ on behalf of the Daya Bay collaboration}

${ }^{a}$ University of California in Irvine, Department of Physics \& Astronomy, 4129 Frederick Reines Hall, Irvine, California, USA

E-mail: broskove@uci.edu

In 2012, the Daya Bay experiment made the first definitive observation of a non-zero neutrino oscillation parameter $\theta_{13}$ using a sample of antineutrinos where neutrons captured on gadolinium $(\mathrm{nGd})$. Later on, the experiment provided another independent measurement with the sample of neutrons captured on hydrogen $(\mathrm{nH})$. Since the beginning of data taking, the experiment has accumulated nearly 4 million reactor antineutrino candidates in about 2000 days and improved the systematic uncertainties. We present the latest Daya Bay $\mathrm{nGd}$ and $\mathrm{nH}$ neutrino oscillation results, as well as the most recent results of a search for a sterile neutrino.

40th International Conference on High Energy physics - ICHEP2020

July 28 - August 6, 2020

Prague, Czech Republic (virtual meeting)

${ }^{*}$ Speaker 


\section{The Daya Bay Reactor Neutrino Experiment}

The Daya Bay Reactor Neutrino Experiment was designed to conduct a precision measurement of the $\theta_{13}$ mixing angle by studying reactor antineutrino disappearance at a $\sim 2 \mathrm{~km}$ baseline as a result of neutrino oscillation. The experiment is located in the vicinity of the Daya Bay and Ling Ao nuclear power plant complex, about $60 \mathrm{~km}$ northeast from Hong Kong. There are in total six $2.9 \mathrm{GW}_{\text {th }}$ pressurized water reactor cores, which makes it one of the most powerful nuclear reactor sites in the world. Each reactor emits $\sim 6 \times 10^{20} \bar{v}_{e} / \mathrm{s}$ isotropically, serving as an abundant source of pure electron antineutrinos with $\lesssim 10 \mathrm{MeV}$ energy.

The Daya Bay experiment consists of 8 antineutrino detectors (ADs) in 3 underground experimental halls (EHs), as shown in the left panel of Fig. 1. There are two near EHs, each with 2 ADs, located at $\sim 400 \mathrm{~m}$ close to the Daya Bay and Ling Ao nuclear cores to sample the reactor antineutrino flux before any large oscillation effects take place. There is a far experimental hall with 4 ADs located at the optimal distance of $\sim 1900 \mathrm{~m}$ to observe a maximal effect of reactor antineutrino disappearance. The comparison of near-far measurements significantly suppresses those systematic uncertainties that are correlated between ADs, allowing for a very precise measurement of the oscillation parameters.
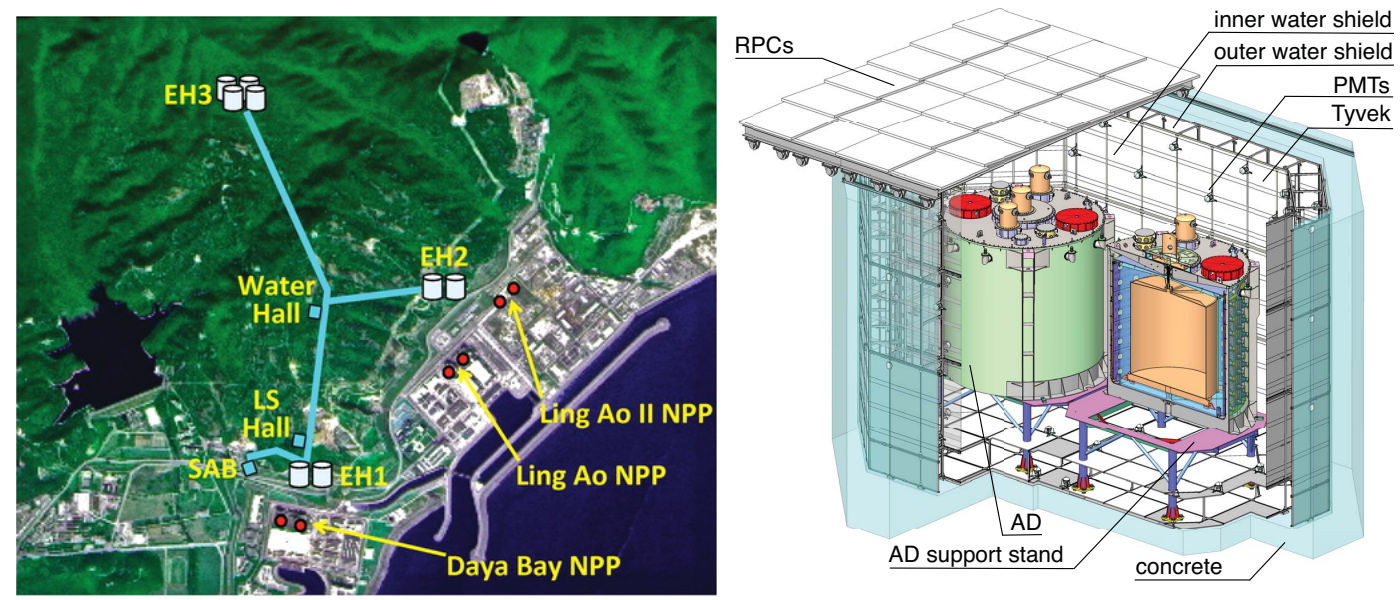

Figure 1: (Left) Layout of the Daya Bay experiment. Antineutrinos from six Daya Bay, Ling Ao and Ling Ao II reactor cores are sampled by two ADs in each of two near experimental halls (EH1 and EH2) while the largest oscillation effect is observed by 4 ADs placed in the far experimental hall (EH3). (Right) Section of the detectors in one of the near halls. The ADs are immersed in a pool of ultra pure water that is covered with RPCs. Section of one of the ADs shows the segmentation into three volumes.

ADs are submerged in instrumented pools of ultra pure water, as shown in the right panel of Fig. 1. The water pool provides passive shielding against cosmogenic neutrons and ambient radioactivity and serves as an active Cherenkov detector for cosmic-ray muon tagging. The water pools are covered by an array of 4-layer Resistive Plate Chambers (RPCs) for further improvement of muon detection. The ADs consist of three nested cylindrical volumes separated by two concentric transparent acrylic vessels. The innermost volume contains $20 \mathrm{t}$ of gadolinium-doped liquid scintillator (LS) serving as a main target. The middle volume holds $22 \mathrm{t}$ of pure LS that detects 
escaping gammas from the central volume of the detector. And the outermost volume is filled with non-scintillating mineral oil working as a buffer zone. The most outer volume also hosts 192 inward-facing 8-inch photomultiplier tubes to collect the scintillation light.

Reactor antineutrinos are detected via the inverse beta decay (IBD) reaction: $\bar{v}_{e}+p \rightarrow e^{+}+n$. The incoming antineutrino interacts with a free proton, naturally abundant in organic LS, creating a positron and a neutron. The positron quickly looses its energy and annihilates while the neutron takes some time to thermialize and is captured on gadolinium ( $\mathrm{nGd}$ ) yielding a $\sim 8 \mathrm{MeV}$ gamma cascade, or on hydrogen $(\mathrm{nH})$ producing a single $2.2 \mathrm{MeV}$ gamma. The spatial $(\lesssim 50 \mathrm{~cm})$ and temporal ( $\sim 30 \mu \mathrm{s}$ for $\mathrm{nGd}, \sim 200 \mu \mathrm{s}$ for $\mathrm{nH}$ ) coincidence of the prompt positron signal and the delayed neutron capture allows for a powerful background rejection. The energy of the prompt signal can be directly related to the incoming antineutrino energy as $E_{\bar{v}_{e}} \simeq E_{\text {prompt }}+0.78 \mathrm{MeV}$.

\section{Oscillation Results}

Neutrino oscillation, the consequence of mixing between flavor and mass neutrino states, is nowadays a well-established phenomenon. Most measurements, including those from Daya Bay, are consistent with mixing between three active neutrinos. The recent measurement of the reactor antineutrino disappearance at Daya Bay using neutron capture on gadolinium is based on 1958 days of data taking [1]. It uses nearly 4 million IBD candidates with about 0.5 million collected in the far EH, which constitutes the largest data set of reactor antineutrinos in the world, and represents a $\sim 40 \%$ increase in statistics with respect to the previous result [2].

Apart from larger statistics, several major systematic improvements were conducted. The absolute energy response uncertainty was reduced to better than $0.5 \%$ [3] mainly thanks to the installation of a full Flash-ADC readout in one of the ADs, which allowed to decouple the nonlinearity intrinsic to the scintillator from the one in the electronic, and to a special calibration campaign in 2017, during which radioactive sources with different enclosures were deployed to the $\mathrm{AD}$ in order to constrain optical shadowing effects.

Daya Bay is a low-background experiment with a background to signal ratio better than $2 \%$ for the nGd IBD sample. The dominant background uncertainty comes from the $\beta$-n decays of cosmogenically produced isotopes, such as ${ }^{9} \mathrm{Li}$ and ${ }^{8} \mathrm{He}$. Better separation of IBDs and ${ }^{9} \mathrm{Li} /{ }^{8} \mathrm{He}$ decays was achieved by increasing the prompt energy cut in the background rate estimation analysis, feasible only due to large statistics. The cleaner sample of cosmogenic isotope decays led to the reduction of this background rate uncertainty from $50 \%$ to $30 \%$.

Finally, the uncertainty on the antineutrinos from spent nuclear fuel, which contributes to $\sim 0.3 \%$ to the total antineutrino signal and was previously conservatively estimated to be $100 \%$, was reduced to $30 \%$ by a careful investigation of its history in collaboration with the nuclear power plant [4].

A relative comparison of the rate and energy spectrum shape of the reactor antineutrinos detected across all ADs, shown in left panel of Fig. 3, yielded $\sin ^{2} 2 \theta_{13}=0.0856 \pm 0.0029$ and $\Delta m_{e e}^{2}=\left(2.522_{-0.070}^{+0.068}\right) \times 10^{-3} \mathrm{eV}^{2}$, where $m_{e e}^{2}$ is an effective mass-squared difference independent on neutrino mass ordering [5]. The best fit with the confidence regions is shown in the right panel of Fig. 3. Likewise, a value of $\Delta m_{32}^{2}=\left(2.472_{-0.070}^{+0.068}\right) \times 10^{-3} \mathrm{eV}^{2}$ was obtained assuming 
the normal ordering and $\Delta m_{32}^{2}=-\left(2.575_{-0.070}^{+0.068}\right) \times 10^{-3} \mathrm{eV}^{2}$ assuming the inverted ordering. This represents the world's most precise estimate of $\sin ^{2} 2 \theta_{13}$. Daya Bay is expected to further improve this measurement and achieve $<3 \%$ precision with the final data set, when the experiment stops data taking at the end of 2020. This will be the most precise measurement of this parameter for the foreseeable future. Similarly, $\Delta m_{32}^{2}$ is also measured with a leading precision, which is comparable to that achieved by accelerator experiments.
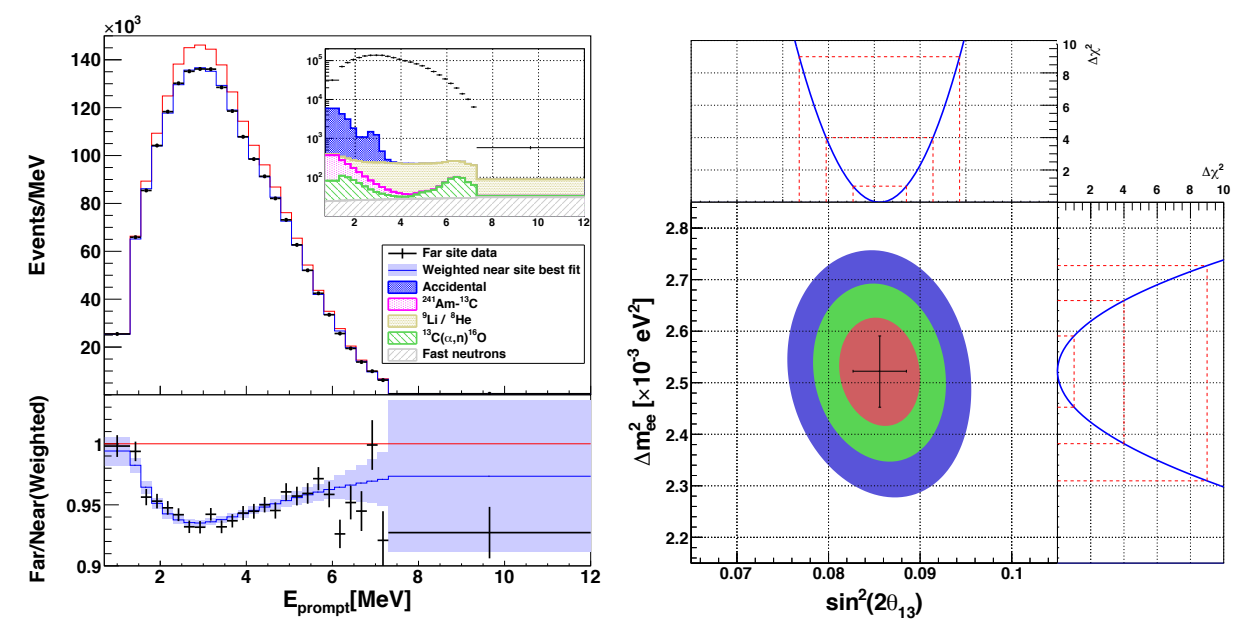

Figure 2: (Left) Energy spectrum of reactor antineutrinos detected in the far hall ADs (black points) is compared to the prediction based on the near halls data assuming no oscillations (red line) resulting in the best oscillation fit (blue line). (Right) The best fit of $\sin ^{2} 2 \theta_{13}$ and $\Delta m_{e e}^{2}$ and the $1,2,3 \sigma$ confidence regions.

An alternative measurement of the $\theta_{13}$ mixing angle was performed, based on 621 days of data taking, using an IBD sample identified via neutron capture on hydrogen [6]. This measurement is largely independent of the $\mathrm{nGd}$ measurement, since the samples are completely different in statistics and mostly decoupled in systematic uncertainties. However, the analysis poses more challenges due to the larger systematics, particularly from the larger background dominated by the accidental coincidence of two uncorrelated signals (the background to signal ratio reaches 1 for the far $\mathrm{EH}$ ADs). A comparison of relative rates results in $\sin ^{2} 2 \theta_{13}=0.071 \pm 0.011$. A spectral analysis is under preparation.

\section{Search for Sterile Neutrino Mixing}

Despite the success of the three-neutrino mixing model, several experimental anomalies can be explained by the existence of sterile neutrino(s) [7]. Daya Bay searched for light sterile neutrino mixing within the minimal extension 3 (active) +1 (sterile) neutrino model. With its unique layout with multiple baselines, the experiment can explore several orders of $\left|\Delta m_{41}^{2}\right|$. The existence of an extra neutrino could appear in the data as an additional spectrum distortion driven by the new mass-squared difference $\Delta m_{41}^{2}$. However, no deviation from the three-neutrino model was found analyzing a data set acquired over 1230 days [8]. Thus Daya Bay was able to place limits on sterile neutrino mixing $\sin ^{2} 2 \theta_{14}$ for three orders of magnitude in $\left|\Delta m_{41}^{2}\right|$, yielding the most stringent 
constrains up to date for $\left|\Delta m_{41}^{2}\right|<0.2 \mathrm{eV}^{2}$, as shown in the left panel of Fig. 3 .
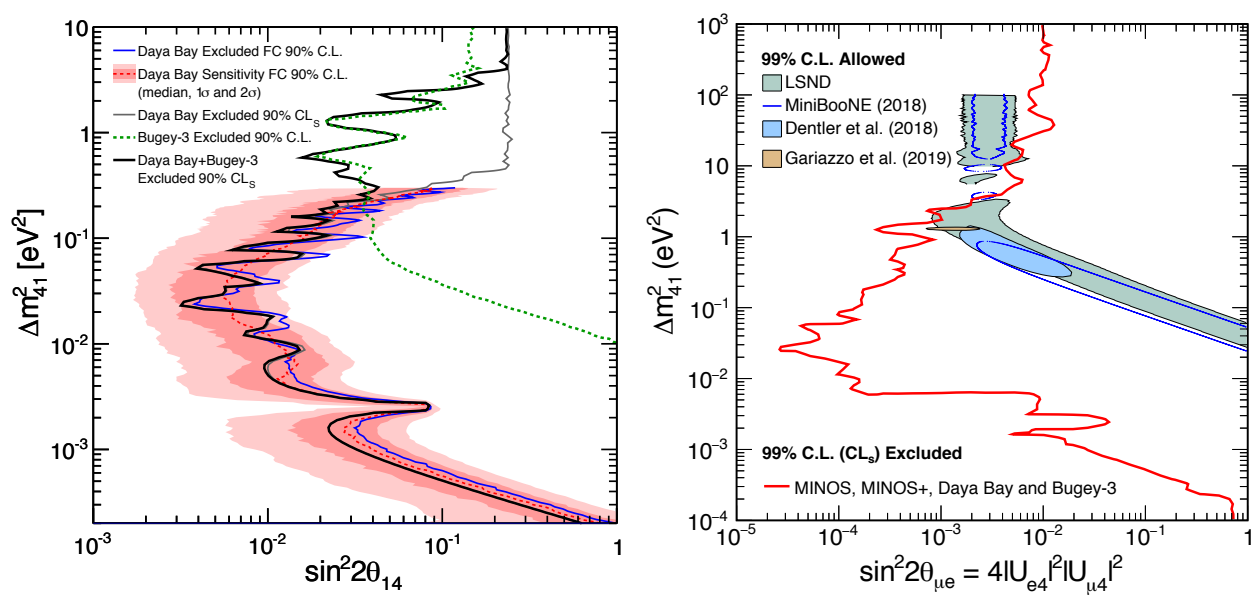

Figure 3: (Left) Exclusion region at 90\% C.L. from Daya Bay obtained with the Feldman-Cousins (red) and $\mathrm{CL}_{s}$ (gray) statistical methods, and its extension with the Bugey-3 data (black) (Right) Exclusion region at 99\% $\mathrm{CL}_{s}$ from the combination of the Daya Bay, Bugey-3, MINOS and MINOS+ experiments, together with the $99 \%$ C.L. allowed region from the LSND and MiniBooNE experiments, is shown. A global fit $[9,10]$ as well as a fit to appearance experiments only [11] is ruled out at $>99 \%$ C.L. The regions to the right of the curves are excluded.

This measurement was extended by the limits from Bugey-3 and further combined with the results from MINOS and MINOS+ experiments to scrutinize the allowed sterile neutrino parameter space of the LSND [12] and MiniBooNE [13] experiments. The excess of electron (anti)neutrinos in the muon (anti)neutrino beam might be explained by sterile neutrino mixing with $\Delta m_{41}^{2} \sim 1 \mathrm{eV}^{2}$ and an effective mixing angle $\sin ^{2} 2 \theta_{\mu e} \equiv \sin ^{2} 2 \theta_{14} \sin ^{2} \theta_{24}$. The $\theta_{14}$ and $\theta_{24}$ mixing angles can be directly constrained by the electron and muon (anti)neutrino disappearance experiments respectively, hence the combination of Daya Bay and Bugey-3 with MINOS and MINOS+. Since no deviation from a three-neutrino paradigm was observed, the combination yielded strong constrains on the sterile neutrino mixing over seven orders of magnitude in $\Delta m_{41}^{2}$. The LSND and MiniBooNE $99 \%$ C.L. allowed regions were excluded at $99 \% \mathrm{CL}_{s}$ for $\Delta m_{41}^{2}<1.2 \mathrm{eV}^{2}$, as shown in right panel of Fig. 3, suggesting that excess in those experiments might not be caused by sterile neutrinos. Furthermore, global fit to the experiments searching for sterile neutrinos $[9,10]$ as well as fit only to the appearance experiments [11] were fully excluded on more than $99 \%$ C.L..

\section{Summary}

The recent measurement of reactor antineutrino oscillation at Daya Bay experiment provides the most precise determination of the $\theta_{13}$ mixing angle and an estimate of $\left|\Delta m_{32}^{2}\right|$ with competitive precision to accelerator experiments. A consistent estimate of $\theta_{13}$ is yielded from an independent measurement using a sample using neutron capture on hydrogen. An improved search for light sterile neutrino mixing results in the world's most stringent limits for $\left|\Delta m_{41}^{2}\right|<0.2 \mathrm{eV}^{2}$. The combination of Daya Bay and Bugey-3 with MINOS and MINOS+ ruled out a significant part of 
allowed sterile neutrino parameter space of the LSND and MiniBooNE experiments. The Daya Bay experiment will continue to take data until the end of 2020, expecting to push the $\theta_{13}$ uncertainty bellow $3 \%$.

\section{References}

[1] Daya Bay collaboration, Measurement of the Electron Antineutrino Oscillation with 1958 Days of Operation at Daya Bay, Phys. Rev. Lett. 121 (2018) 241805 [1809. 02261].

[2] DAYA BAY collaboration, Measurement of electron antineutrino oscillation based on 1230 days of operation of the Daya Bay experiment, Phys. Rev. D 95 (2017) 072006 [1610.04802].

[3] DAYA BAy collaboration, A high precision calibration of the nonlinear energy response at Daya Bay, Nucl. Instrum. Meth. A 940 (2019) 230 [1902 .08241].

[4] X. Ma, Y. Zhao, Y. Chen, W. Zhong and F. An, Antineutrino flux and spectrum calculation for spent nuclear fuel for the Daya Bay antineutrino experiment, Nucl. Phys. A 966 (2017) 294 [1512.07353].

[5] Daya Bay collaboration, New Measurement of Antineutrino Oscillation with the Full Detector Configuration at Daya Bay, Phys. Rev. Lett. 115 (2015) 111802 [1505.03456].

[6] DAYA BAY collaboration, New measurement of $\theta_{13}$ via neutron capture on hydrogen at Daya Bay, Phys. Rev. D 93 (2016) 072011 [1603.03549].

[7] S. Böser, C. Buck, C. Giunti, J. Lesgourgues, L. Ludhova, S. Mertens et al., Status of Light Sterile Neutrino Searches, Prog. Part. Nucl. Phys. 111 (2020) 103736 [1906. 01739].

[8] MINOS+, DAya BAy collaboration, Improved Constraints on Sterile Neutrino Mixing from Disappearance Searches in the MINOS, MINOS+, Daya Bay, and Bugey-3 Experiments, Phys. Rev. Lett. 125 (2020) 071801 [2002.00301].

[9] S. Gariazzo, C. Giunti, M. Laveder and Y. Li, Updated Global 3+1 Analysis of Short-BaseLine Neutrino Oscillations, JHEP 06 (2017) 135 [1703.00860].

[10] S. Gariazzo, C. Giunti, M. Laveder and Y. Li, Model-independent $\bar{v}_{e}$ short-baseline oscillations from reactor spectral ratios, Phys. Lett. B 782 (2018) 13 [1801.06467].

[11] M. Dentler, A. Hernández-Cabezudo, J. Kopp, P.A. Machado, M. Maltoni, I. Martinez-Soler et al., Updated Global Analysis of Neutrino Oscillations in the Presence of eV-Scale Sterile Neutrinos, JHEP 08 (2018) 010 [1803. 10661].

[12] LSND collaboration, Evidence for neutrino oscillations from the observation of $\bar{v}_{e}$ appearance in a $\bar{v}_{\mu}$ beam, Phys. Rev. D 64 (2001) 112007 [hep-ex/0104049].

[13] MiniBooNE collaboration, Significant Excess of ElectronLike Events in the MiniBooNE Short-Baseline Neutrino Experiment, Phys. Rev. Lett. 121 (2018) 221801 [1805. 12028]. 